\title{
Design of E-commerce Data Scalable Storage System Based on Mobile Internet Communication Technology
}

\author{
Jiejie Cui ${ }^{1}$, Xiang $\mathrm{Li}^{2}$, Yang Wang ${ }^{3 *}$ \\ ${ }^{1}$ Department of International Law Affairs, Graduate School, Dong-A University \\ Busan, Korea \\ ${ }^{2}$ School of Chemistry and Chemical Engineering, Chongqing University of Technology \\ Chongqing 400054, China \\ ${ }^{3}$ Chongqing Intellectual Property School, Chongqing University of Technology \\ Chongqing 400054, China \\ *Corresponding author: midq8th@163.com
}

Received: July 27, 2021. Revised: December 18, 2021. Accepted: January 13, 2022. Published: January 14, 2022.

\begin{abstract}
The traditional encrypted storage system is inefficient when it encrypts the data of the Internet of Things, and there are few IOT data nodes that can be encrypted in a short time. In order to solve the above problems, a new Internet of Things data effective information encryption storage system is proposed. The hardware and software of the system are mainly designed. The chip selected for the collector is TTSAD251, which can expand the collection range. The processor is set with multiple cores to reduce the system power consumption. The memory uses SPRTAN-2 chip as the structure chip. The software work consists of three parts: collecting effective information of Internet of Things big data, establishing encrypted documents and storing effective information of big data of Internet of Things. In order to detect the working effect of the system, the experimental comparison with the traditional system shows that the proposed encryption storage system can improve the storage range of big data effective information of the Internet of Things by $\mathbf{2 0 . 5 8 \%}$, and the work efficiency by $5.64 \%$. Compared with the traditional system, the designed system also has obvious advantages in the number of big data node secrets. In different files, the average number of big data information node encryption in this system is about 166,700 . The experimental data show that the designed system has ideal application performance and provides a reliable basis for related fields.
\end{abstract}

Keyword-Mobile Internet; Communications technology; Electronic Commerce; Big data expansion; Storage system.

\section{INTRODUCTION}

With the upgrading of the network, big data is constantly impacting people's lives. People's dependence on the Internet is increasing, and the demand for Internet information quality is increasing. In the era of wide dissemination of information, big data security has become one of the issues that people attach great importance to, and big data information storage has naturally become the top priority of network information system [1].

In this situation, the traditional big data storage system focuses on the setting of big data security and peripheral protection equipment and password security, which is basically the surface business system facility management. Although the security equipment plays an important role in network malicious attacks, the demand for big data storage is increasing, and once the storage medium is damaged, it will cause big data loss. The real network threat is not malicious trojan virus and network hacker attacks, but computer terminal system information and internal control. Therefore, encryption technology for basic big data storage protection is particularly important [2].

Most of the departments, enterprises and institutions store the big data directly in the storage medium. Once the basic hardware is moved or damaged, the big data will be destroyed, causing unspeakable disaster to their work. The existing security system focuses on the system configuration, the operation is complex, the application is not wide, and the encryption system is not perfect, which has a great impact on users. In the information security system, with the development of information technology and Internet, the demand for security standards is constantly improving, and the solution of big data security is also a gradual process. Information security includes the integrity, stable storage, controllability and availability of information. Information security system is 
a huge and complex system. In order to improve the system security more effectively, we should integrate the system management and administrative management, and work together. At the same time, we should ensure the agility of the system, make the important big data transmission complete, and protect the confidentiality of users.

Reference [3] proposed a layered extensible storage architecture for massive electronic credential, combined with hash mode-taking algorithm and consistent hash algorithm to achieve fast data location, and designed a horizontal expansion scheme based on hash mode-taking algorithm to reduce the amount of data migrated during node addition and deletion. In addition, a cache scheme based on hot data and a load balancing scheme based on access delay are designed and implemented to further improve the speed of data access. Reference [4] proposed a multi-copy generation and corrupted data recovery algorithm based on multi-level network coding. Based on multi-level network coding, the algorithm improves the coding matrix of erasable codes to form a multilevel coding matrix, and generates hierarchical coding ( $\mathrm{HC}$ codes) to form multi-copy data by utilizing its cascades, so that there are coding relationships among copies. In case of damaged data recovery, the data encoding information provided by the data owner and data blocks stored in the cloud storage are used to directly calculate the data recovery, thus avoiding remote data download from the cloud storage. However, the above literature method is inefficient in the encryption of iot data, and there are few iot data nodes that can be encrypted in a short time.

In order to ensure the safe storage of big data, this paper proposes a scalable storage system of e-commerce big data based on mobile Internet communication technology. The hardware and software of the system are optimized. The system has a network system with higher storage and storage security.

\section{HARDWARE DESIGN OF E-COMMERCE BIG DATA}

\section{SCALABLE STORAGE SYSTEM BASED ON MOBILE INTERNET}

\section{COMMUNICATION TECHNOLOGY}

In the environment of Internet of Things, there is a large number and variety of big data effective information, so the design of encrypted storage system is required to have fast collection speed and large capacity. The hardware structure of the e-commerce big data scalable storage system based on mobile internet communication technology is shown in Figure 1:

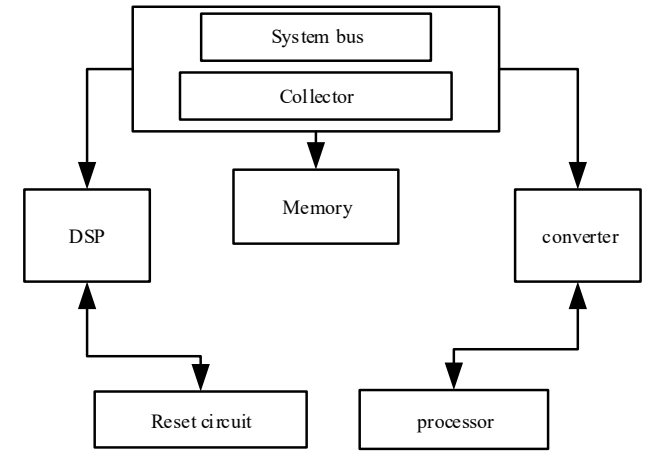

Figure 1. Hardware structure of encrypted storage system

It can be seen from Figure 1 that the designed big data effective information encryption storage system of Internet of Things is mainly composed of system bus, collector, memory, DSP, converter, reset circuit and processor [5]. The hardware circuit diagram of the storage system is shown in Figure 2:

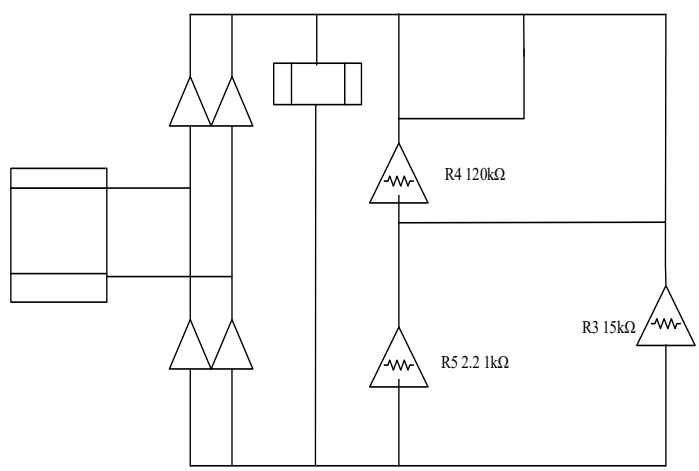

Figure 2. Hardware circuit diagram

In this paper, the collector, processor and memory are designed. The design process is as follows.

\section{A. Collector design}

The internal chip of the collector studied in this paper is TTSAD251 chip produced by Danish company. The collector is installed in the embedded processing unit. This unit is the main unit designed in this paper, which is mainly responsible for collecting information and management, sending big data to the storage unit and display unit regularly, parsing user instructions, completing the collector maintenance commands and executing its daily collection work [6]. The big data received by the remote big data collector is sent to the information processor, and the high-speed network interface and other network interfaces are used for big data exchange, and the monitoring big data and information are shared. The acquisition interface can provide instructions and corresponding services, meeting the requirements of local and remote system acquisition [7]. The internal structure of the collector is shown in Figure 3: 


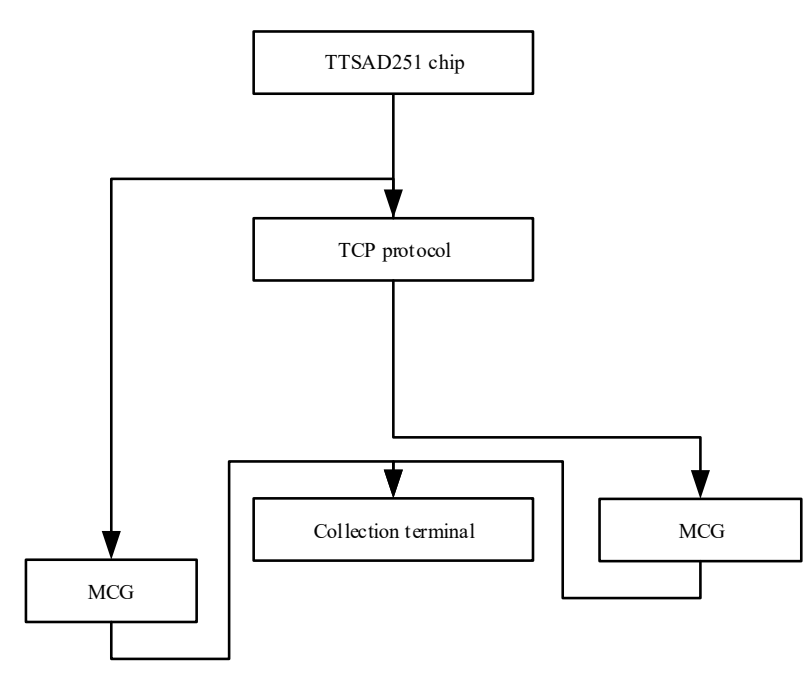

Figure 3. Structure design of collector

This system uses RTU type collector, which is a multi-functional big data monitoring and acquisition terminal. It can realize remote terminal management, monitoring and setting through mobile terminal and computer interface, so as to achieve a large number of effective information collection of Internet of Things. The collector has high-end communication module and integrates 64 bit stable and reliable high-performance processor MCG, which is different from the traditional system. This paper uses embedded real-time operating system, which can provide multi-channel industrial standard transmitter, thermal resistance input, multi-channel digital input and multi-channel output [8].

The big data collector works in the FPGA center, and uses the timing control of the control unit to collect, store and edit analog signals.

When the big data collector receives the "start" command, the power system will automatically transmit electricity to the collector, so that the system can always maintain the voltage above $5 \mathrm{~V}$. The collector is equipped with a signal conditioning module to reduce the received voltage to $0 \mathrm{~V}-3.3 \mathrm{~V}$ to prevent the input impedance of the acquisition device. In the process of collecting network sensitive big data, foreign signals will continue to invade, so the system has set up 12 conversion channels, 24 bit $\mathrm{A}$ / D conversion system, and the central control module controls the internal operation logic. The collector can collect up to $128 \mathrm{M}$ network sensitive big data and store them in the memory at the same time.

The big data collector designed in this paper has high intelligence. After the central power supply is cut off, the collector can also maintain normal work to prevent big data leakage and untimely storage. The capacity of the central control big data collector is more than 8 bits. The memory itself has the function of protecting big data, so there is no power failure protection big data in the collector. This design method makes the big data collected far meet the required storage capacity.

The collector itself has high requirements for power supply, and the design principle always follows the principle of low power consumption. The power supply chip is MAX888 control chip, which can reduce the input voltage by half and the power consumption of the system is analyzed by the logic control center to ensure the normal power supply of the system.

This collector is suitable for all kinds of wired and wireless networks. It can be configured according to its own needs. After triggering, it will inform the user in time, and transmit the big data to each monitoring center, and inform that it can perform the switching action of some devices [9]. As a remote control acquisition terminal, RTU is suitable for all kinds of mobile networks in China, and is widely used in current, voltage, pressure, agriculture, warehouse, computer room and other places in industrial field. At the same time, the collector set in this paper increases the keyless opening function, so that the user can open the function within the effective time, and the work is more flexible. Powerful programmable ability can be used as remote editing, convenient to collect information, users can also through SMS, network background and other big data collection on the current device, monitoring the implementation of action. The RTU collector supports all TCP protocols without second development.

\section{B. Processor design}

At present, the commonly used processor is single core processor, but the parallel instructions and parallel big data of single core processor are difficult to be improved, and the running process frequency is high, which will lead to a great increase in processor power consumption [10]. Now the only solution is to increase the number of cores. The more cores there are, the more instructions can be executed, so as to achieve high efficiency. This paper uses a multi-core processor with IDS interface, memory controller and PCI-E interface. The structure of the processor is shown in Figure 4:

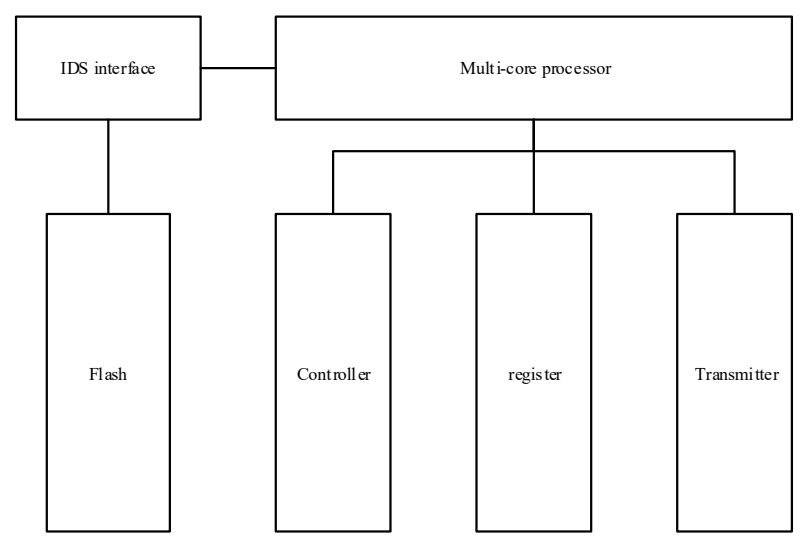

Figure 4. Processor architecture

Because there are many cores in the system, there must be some problems such as task allocation, scheduling and balance. Scheduling is the core of the whole processor. The current system can not support the operation of multi-core processor. In order to meet the processing requirements, the load must be balanced. In this paper, three multi-core scheduling algorithms are added to the processor, which are global queue scheduling, symbiotic 
queue scheduling and local queue scheduling [11]. The global queue scheduling algorithm is mainly to maintain the global tasks and wait for the queue. When the kernel is idle, the global tasks will select the ready tasks to execute in this core. Its advantage is that the kernel utilization rate is very high. The advantage of local queue scheduling algorithm is that it does not need to switch CPU, and can improve the hit rate of local cache. Its disadvantage is also obvious, the CPU hit rate is very low. Symbiotic scheduling algorithm is to schedule more tasks and less tasks together to reduce resource conflicts. At present, global queue scheduling algorithm is widely used. The internal circuit diagram of the processor is shown in Figure 5:

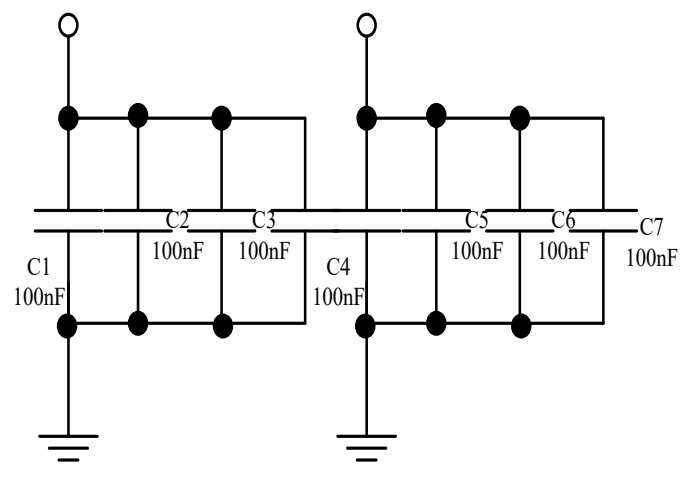

Figure 5. Processor circuit diagram

This paper also uses the principle of balanced design to balance the internal aspects of the chip, so that the processor will not lose the balance of other aspects in order to obtain one aspect. In the process of running, CPU will weigh the solution of the problem and adopt a simple, easy to implement and low-cost design. The structure design is mainly aimed at the whole. In general, the simple logic of the processor can play a good role for most applications. In order to achieve the overall goal, we have to sacrifice one side. In multi-core processor, we should take scientific analysis results as the basis, adhere to the principle of balance, and ensure the whole operation of the system.

\section{Memory design}

In this paper, the memory is based on SPRTAN-2 chip. The memory structure is composed of three levels: distributed memory structure, block memory structure and ISHR interface with external memory. The memory structure is shown in Figure 6:

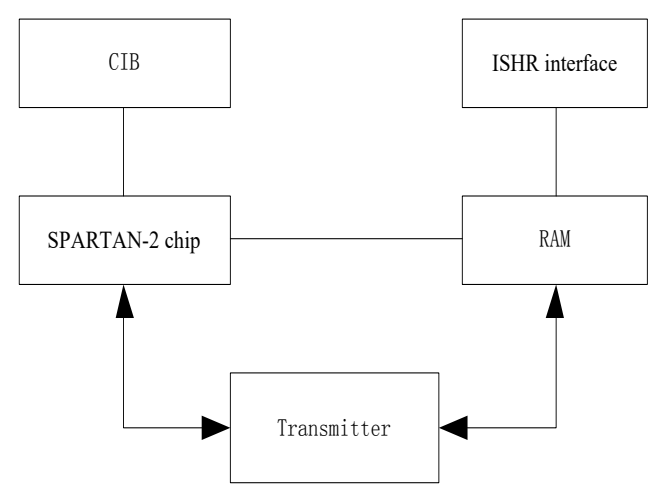

Figure 6. Memory structure

Each CIB in the distributed memory can form two $72 \times$ 1 or $1 \mathrm{ZJ} \times 4$ processors, which have the advantages of large memory and fast access speed. Therefore, it is usually used for processing big data cache, and the memory can be configured with dual port RAM. If it is converted to large memory, multiple CIBs must be connected, which will greatly reduce the access speed and occupy logical resources. Block memory FIPA is a special device, usually installed next to the chip and arranged vertically [12]. SPRTAN-2 chip has multi block memory structure, each block accounts for $28 \mathrm{~Kb}$, the maximum limit can provide $1401.5 \mathrm{Mb}$ block memory, different models, the number and proportion are also different. The memory can be configured with single port BLAY ram or dual port BLAY ram. In addition to single and dual port memory, block memory can also convert the width of big data, so that multiple BLAY RAM can be combined to become a larger storage unit.

When reading / writing the same BLAY RAM address, it can be read through the following three processing modes: write priority mode, read priority mode and output invariant mode, and can be designed by ISE design tool. In the write priority mode, read / write the same address in the BLAY RAM at the same time, and read / write the same big data; in the read priority mode, read / write the same address in the BLAY RAM at the same time, and read the big data in the buffer first, which does not affect the write operation; in the output invariant mode, the same address in the BLAY RAM is read/ written at the same time. The big data is written into the buffer without affecting the output, and the last read operation big data is kept. High speed access to external memory can be realized by using ISHR interface and DOM interface. These interfaces include SSIL-I, SSIL-T, SKV-24 / I / II, and some DRI functions in IIB module. DILIN software provides a lot of reference designs for high speed memory interface [13-14].

WFS cloud storage is selected for big data storage, and WEB service configuration interface is set to manage network sensitive big data and complete system storage and application. When big data storage stores, searches and shares all kinds of files, users can use the virtual space as the security space, and set various parameters in the virtual space. There are many distributed files in the big data memory. Each distributed file can be used for secondary development of network sensitive big data. Users do not need to spend time to record how it is implemented after designing big data parameters.

All resources of big data storage are described by cloud storage. Network sensitive big data and big data are stored separately. When external users access resources, they must join a third party to store big data. The file name in cloud storage system should record the type, size and storage date of network sensitive big data in detail, verify the storage effect through Da algorithm, and record the hash value of storage. The memory needs to be stored in batches, and all the folders will be backed up in the URI, so as to provide services to the outside world anytime and anywhere. 
The memory designed in this paper can copy, cut and delete network sensitive big data independently. The operation does not need to extract all the big data, only need to update the big data records, so as to improve the operation efficiency of big data storage. When the hash value of big data is recorded, the copy is consistent with the uploaded big data, and there is no big data redundancy in the system [15]. Big data storage has multiple cloud storage interfaces, which can be accessed through Http and Https. At the same time, it also has API access interface to control external big data intrusion and effectively ensure big data security.

The operation circuit diagram of big data memory is shown in Figure 7:

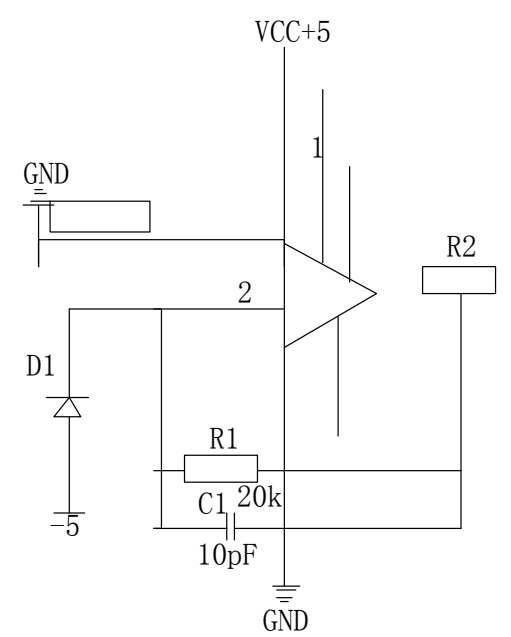

Figure 7. Circuit diagram of big data memory

It can be seen from the above figure that the big data memory has a total of 12 power resistors, which effectively controls the operation of the system. When the memory starts to work, every part of the circuit will work automatically to improve the storage efficiency and ensure that the storage process will not be invaded by external signals [16-17].

\section{SOFTWARE DESIGN OF E-COMMERCE BIG DATA}

\section{SCALABLE STORAGE SYSTEM BASED ON MOBILE INTERNET}

\section{COMMUNICATION TECHNOLOGY}

According to the designed hardware of e-commerce big data scalable storage system of mobile internet communication technology, the software is designed, and the software work flow chart is constructed, as shown in the Figure 8:

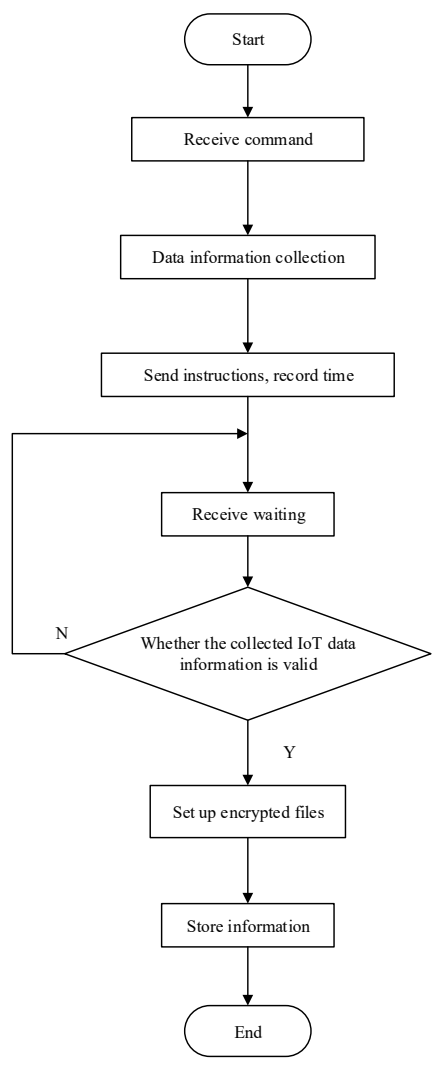

Figure 8. Flow chart of storage system software

Analysis of the above figure shows that the design of mobile internet communication technology e-commerce big data scalable storage system is divided into three steps [18-19].

The first step: collect effective information of Internet of Things big data. There are many kinds of big data information in the Internet of Things, and they are used for different units. Some big data information is suitable for enterprises, some for individuals, and some for governments. Therefore, the collected information should be classified and processed.

The second step: create an encrypted document. Encrypted documents will not affect users' use of other devices. The internal security environment has the advantages of simple structure, convenient management and strong confidentiality. According to different encryption methods, encrypted documents can be set into two modes: network version and stand-alone version [17]. The network version refers to work in the network environment, in addition to the ability to complete the storage work, but also to complete the tracking service; the stand-alone version refers to the encrypted document that is not connected to the network, and the encrypted file only completes the encryption of the effective information of the machine [20-21].

The third step: the Internet of Things big data effective information storage. In order to improve the security of storage, all the stored documents should be set with access rights, and the stored procedure should be set to be completely transparent to ensure that users can use the storage device smoothly in the shared or offline mode. It is forbidden for applications to print, steal and drag stored big data unless they have access rights. The stored files should be regularly audited and managed by professional 
management personnel. The client should set double factor authentication to ensure that the client will not be damaged by external information, and the system will always work in a safe and reliable state.

\section{VERIFICATION EXPERIMENT}

\section{A. Purpose of the experiment}

In order to test the actual effect of the e-commerce big data scalable storage system based on mobile internet communication technology, compared with the traditional system, the encryption effect and storage capacity are analyzed.

\section{B. Experimental parameter setting}

The experimental parameters are set as shown in Table 1:

Table 1. Experimental parameters

\begin{tabular}{cccc}
\hline Parameter & $\begin{array}{l}\text { Maximu } \\
\mathrm{m}\end{array}$ & $\begin{array}{l}\text { Average } \\
\text { value }\end{array}$ & $\begin{array}{l}\text { Minim } \\
\text { um }\end{array}$ \\
\hline Input paranoid current & $23 \mu \mathrm{A}$ & $20 \mu \mathrm{A}$ & $15 \mu \mathrm{A}$ \\
Input impedance & $15 \Omega$ & $13 \Omega$ & $7 \Omega$ \\
Input voltage & $150 \mathrm{~V}$ & $100 \mathrm{~V}$ & $45 \mathrm{~V}$ \\
Open loop voltage gain & $70 \mathrm{~dB}$ & $65 \mathrm{~dB}$ & $40 \mathrm{~dB}$ \\
Common mode rejection & $100 \mathrm{~dB}$ & $50 \mathrm{~dB}$ & $25 \mathrm{~dB}$ \\
ratio & $120 \mathrm{~V}$ & $80 \mathrm{~V}$ & $50 \mathrm{~V}$ \\
Rated output voltage & $130 \mathrm{~A}$ & $50 \mathrm{~A}$ & $20 \mathrm{~A}$ \\
Rated output current & $2 \mathrm{~A}$ & $1.5 \mathrm{~A}$ & $0.3 \mathrm{~A}$ \\
Short-circuit current & $18 \Omega$ & $13 \Omega$ & $7 \Omega$ \\
Output resistance & $15 \mathrm{~V}$ & $12 \mathrm{~V}$ & $8 \mathrm{~V}$ \\
Supply voltage & $0.5 \mathrm{~A}$ & $0.3 \mathrm{~A}$ & $0.2 \mathrm{~A}$ \\
Quiescent current & & & \\
\hline
\end{tabular}

\section{Experimental results and analysis}

According to the above parameters, this paper selects the e-commerce big data scalable storage system and traditional system based on mobile internet communication technology to store the same big data effective information at the same time, and records the storage capacity and encryption amount of the two systems at the same time. The experimental results are as follows:

(1) Experimental results of encryption quantity. As is shown in Figure 9:

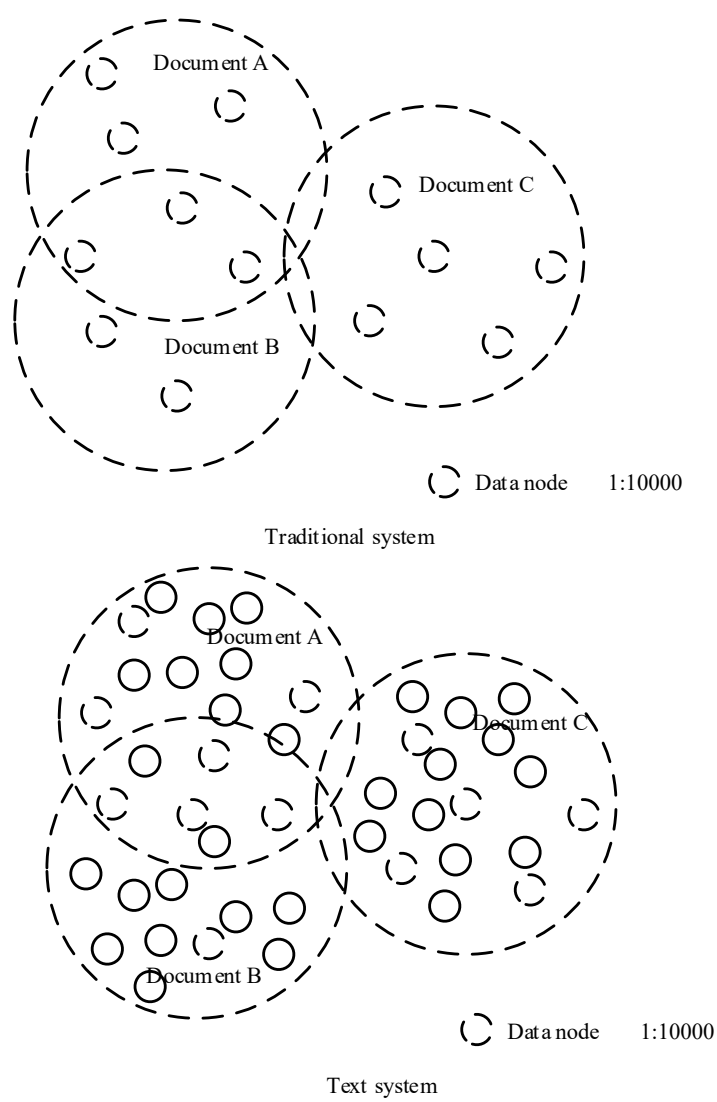

Figure 9. Comparison of encrypted big data experiment

At the ratio of 1:1000, the effective information of the same IOT big data is encrypted at the same time. It can be seen from Figure 9 that the nodes encrypted in this system are much higher than those encrypted in traditional systems. In the three encrypted documents, this system and the traditional system show different encryption capabilities. In document A, the traditional system can only encrypt 50000 big data information nodes, while this system can encrypt 170000 big data information nodes; in document $\mathrm{B}$, the traditional system can only encrypt 20000 big data information nodes, while this system can encrypt 160000 big data information nodes; in document $\mathrm{C}$, the traditional system can encrypt 50000 big data information nodes, while this system can encrypt 170000 big data information nodes.

(2) Experimental results of storage capacity. As is shown in Figure 10: 


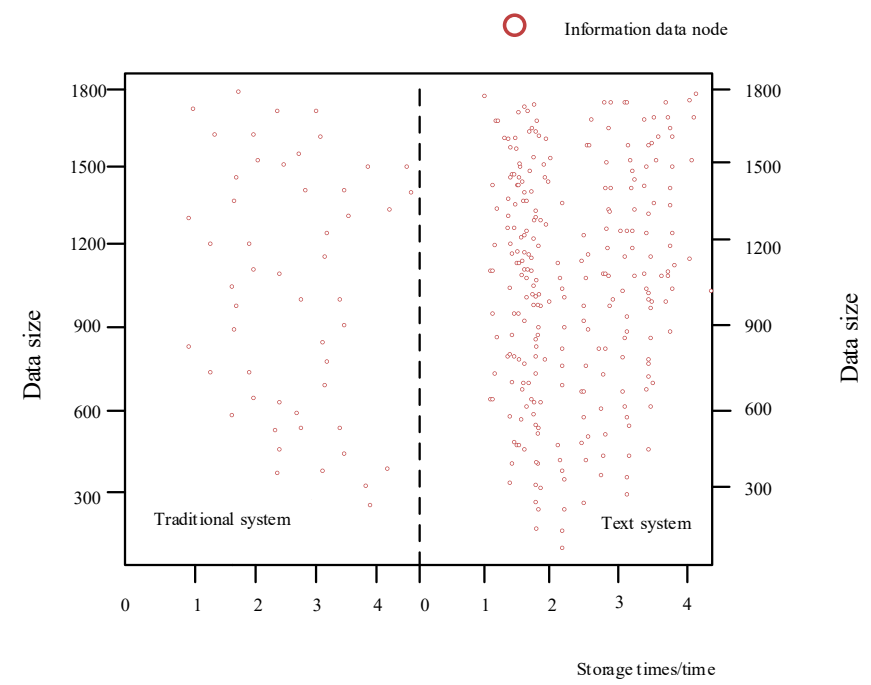

Figure 10. Comparison results of storage capacity experiment

It can be seen from Figure 10 that the system studied in this paper can store more effective information nodes of big data of Internet of Things than traditional systems. Under the same storage times, the number of big data information nodes in the storage system studied in this paper is 3-5 times of the traditional system, and the storage effect is excellent. With the increase of storage times, this advantage is more and more obvious. In the later stage, the traditional system cannot work, but this system can still store a large number of big data.

\section{Empirical conclusion}

According to the above experimental results, the following conclusions are drawn: in a certain period of time, the traditional storage system and the storage system studied in this paper can complete the storage encryption work, but the work efficiency of the system studied in this paper is far higher than that of the traditional storage system, whether it is storage work or encryption work. The storage system studied in this paper connects the hardware and software together, so that the system can encrypt and store a large number of effective information big data in a short time, reduce work costs, improve work efficiency, and has a great market development space.

\section{CONCLUSION}

The encrypted storage system designed in this paper can greatly improve the security of the effective information of the big data of the Internet of Things, expand the storage range of the internal structure of the Internet of Things, the storage range of the Internet of Things can be increased by $20.58 \%$, the perfect combination of hardware system and software system greatly improves the big data transmission performance, speeds up the work efficiency of the storage work, and at least improves the work efficiency by $5.64 \%$. Using the designed experiment to verify the system, it is proved that the system has great feasibility. This study can provide strong technical support and guarantee for the further development of the Internet of Things. In document A, the traditional system can only encrypt 50000 big data information nodes, while this system can encrypt 170000 big data information nodes; in document $\mathrm{B}$, the traditional system can only encrypt 20000 big data information nodes, while this system can encrypt 160000 big data information nodes; in document $\mathrm{C}$, the traditional system can encrypt 50000 big data information nodes, while this system can encrypt 170000 big data information nodes.

Although the system designed in this paper has a series of advantages, the software structure is not clear enough. Due to the massive amount of big data information in the Internet of Things, it is very difficult to collect, and some information cannot be preserved for a long time, which needs to be further studied and discussed in the future.

\section{REFERENCES}

[1] Zhou Q, Zhang Z, Wang Y. Research on safety management system optimization of B2C e-commerce intelligent logistics information system based on data cube. Journal of Intelligent and Fuzzy Systems, 2019, (4):1-8.

[2] Zhang Y. Application of improved BP neural network based on e-commerce supply chain network data in the forecast of aquatic product export volume. Cognitive Systems Research, 2019, 57:228-235.

[3] Li F H, Li D Y, Jin W, et al. Hierarchical scalable storage architecture for massive electronic bill. Journal on Communications, 2019, 40(5):79-87.

[4] Xu G W, Shi Ch H, Feng X Y, et al. Multi-Replica Cloud Data Storage Based on Hierarchical Network Coding. Journal of Computer Research and Development, 2021, 58(2):291-304.

[5] Lima Junior M S P dos S, Halapi M P, Udvary E. Design of a real-time indoor positioning system based on visible light communication. Radio Engineering, 2020, 29(3):445-451.

[6] Huang Q, Liu D, Chen Y, et al. Secure free-space optical communication system based on data fragmentation multipath transmission technology. Optics Express, 2018, 26(10):13536.

[7] Yu M, Shang W, Lu H. A design method of the wide-area damping controller of the power system considering the communication time-delay based on an iterative identification. International Journal of Electronics, 2018, 105(7-9):1551-1567.

[8] Song H, Schnieder E. Development and evaluation procedure of the train-centric communication-based system. IEEE Transactions on Vehicular Technology, 2019, 68(3):2035-2043.

[9] Li Q L, Chen D W, Bao Q, et al. Numerical investigations of synchronization and communication based on an electro-optic phase chaos system with concealment of time delay. Applied Optics, 2019, 58(7):1715-1722.

[10]Xiao J, Zhao C, Feng X, et al. Review on the millimeter-wave generation techniques based on photon assisted for the ROF network system. Advances in Condensed Matter Physics, 2020, 2020, (10):1-14.

[11]Feng S, Tan L. Simulation of sports and health big data system based on FPGA and Internet of Things. Microprocessors and Microsystems, 2020:103-116.

[12] Yan G. Simulation analysis of key technology optimization of $5 \mathrm{G}$ mobile communication network 
based on Internet of Things technology. International Journal of Distributed Sensor Networks, 2019, 15(6):145-155.

[13] Sciullo L, Trotta A, Di Felice M. Design and performance evaluation of a LORA-based mobile emergency management system (LOCATE). Ad Hoc Networks, 2020, 96:101993.1-101993.17.

[14] Wenquan J, Hyeun K D. Design and implementation of e-health system based on semantic sensor network using IETF YANG. Sensors, 2018, 18(2):629-631.

[15]Darwish S M, El-Shnawy A G. An intelligent database proactive cache replacement policy for mobile communication system based on genetic programming. International Journal of Communication Systems, 2018:3536-3547.

[16] Li C C, Ji Z S, Wang F, et al. The design of real-time communication system based on RFM and MRG real time for EAST. IEEE Transactions on Plasma Science, 2018, 46(6):2267-2271.

[17]Xie C, Xiao X, Hassan D K. Data mining and application of social e-commerce users based on big data of internet of things. Journal of Intelligent and Fuzzy Systems, 2020, 39(1):1-11.

[18] Akram S, Hussain S, Toure I K, et al. ChoseAmobile: A web-based recommendation system for mobile phone products. Journal of Internet Technology, 2020, 21(4):1003-1011.

[19] Pramono C A, Manurung A H, Heriyati P, et al. Analysis of the influence of entrepreneurship capability, agility, business transformation, opportunity on start-up behavior in e-commerce companies in indonesia during the Covid 19 Pandemic. WSEAS Transactions on Business and Economics, 2021, 18: 1103-1112.

[20]Chernova V Y, Degtereva E A. Forecast of development of the dual-use industrial products market. WSEAS Transactions on Business and Economics, 2020, 17: 987-992.

[21]Eldar A G. Problems of financial and investment support modelling of the regional social and economic development. WSEAS Transactions on Business and Economics, 2020, 17:741-752.

\section{Creative Commons Attribution License 4.0} (Attribution 4.0 International, CC BY 4.0)

This article is published under the terms of the Creative Commons Attribution License 4.0

https://creativecommons.org/licenses/by/4.0/deed.en US 